\title{
Study on Balance Theory in Vocal Music Piano Accompaniment Rong Fu
}

School of Music, Ningxia University, Yinchuan, 750011, China

\author{
Keywords: Vocal music, Piano accompaniment, Balance theory, Application strategy
}

\begin{abstract}
Vocal music piano accompaniment plays an increasingly high role in modern music performance and receives generation attention of all sectors of society. Vocal music piano accompaniment art should be devoted to pursuing balance of various relations. Hence, higher requirements are proposed for the essence of art, such as mastery of details and overall promotion. Thus, balance theory needs to be introduced in vocal music piano accompaniment. This paper summarizes balance theory, analyzes two pairs of relations of balance theory in different vocal music piano accompaniments and proposes specific application strategies of balance theory in vocal music piano accompaniments.
\end{abstract}

\section{Introduction}

In accordance with actual situation, with regard to piano accompaniment and singers' voice, although most people have fully approached vocal music piano accompaniment should be cooperated with singers, and certain balance should be pursued in volume and playing skills. However, very few can accurately explain what the balance is and how to achieve balance. In the process of vocal music piano accompaniment, it is necessary to confirm the problem that how to achieve the balance between piano and signers' voice. Hence, the author studies how to apply balance theory in vocal music piano accompaniment.

\section{Overview of balance theory}

In the view of the author, clearness should be the foundation of balance theory. In the process of vocal music piano accompaniment, the foundation of all voice effects is clearness degree. The unclear music is like driving on the expressway with the eyes covered. More dangerously, vast audiences are in the front. All factors hindering clearness are important content damaging cooperation balance. In the process of vocal music piano accompaniment, both parties should very clearly express the performance of the other party regardless of softly murmuring or singing loudly, and should exactly feel common music development tendency. Clearness not only includes voice clearness, but also covers clearness of emotional expression. In the whole singing process, both piano and singers should accurately and clearly express the emotions in the song. A singer paying attention to vice and emotion impossibly expresses the meaning clearly under unclear accompaniment. So, it is necessary to fully understand piano accompaniment works. The balance theory means vocal music or instrumental music abs piano can clearly and accurately convey respective sound and emotions to appreciators and coordinate with each other. It thus can be seen that both singers and piano accompanists should activity participate in the performance process.

\section{Two pairs of relations of balance theory in vocal music piano accompaniment}

\section{The relationship between the variable and the constant}

In vocal music piano accompaniment, harmonious degree plays a decisive role in general equilibrium of vocal music piano accompaniment. But, for harmonious degree, the key point of voice part problem is to let vocal music piano accompanists carefully study the key points of balance theory. This is because different voice part singers will have different accompaniment requirements in performance. Piano accompanists need to cooperate with diverse singers, so piano accompanists should deal with the relationship between the variable and the constant. Of course, primary and 
secondary problems of voice part allocation should be noticed in piano accompaniment. The most typical point is that, relatively important and key voice parts in piano should be clearly identified and guided in multifarious voice part lines generated by piano and vocal music. Relative to secondary voice part, it just needs to be placed in the harmonic background. Such situation will appear in the climax part of most vocal music works that, high pitch part on the right hand of piano accompanists basically keeps consistent with vocal music melody. Many accompanists form intuition mistake, regard it as an important voice part and create high pitch melody line together with singers, but they neglect the blending feeling for enriching harmony texture. Thus, monotony and unbalance trends often appear in auditory sense. Even piano and vocal music compete for the main role. Hence, piano accompaniment should leave theme of vocal music and choose fill in harmony texture.

\section{The relationship between reason and sensibility}

Comprehensive and comprehensive rational analysis is very necessary for accompaniment. It is also often mentioned in current piano accompaniment teaching. Meanwhile, high-level accompaniment talents should be actively trained. Accompaniment sense generated intangibly and response capacity of vocal music accompanists are greatly correlated. Dynamic unbalance often exists in singing voice and accompaniment. This requires piano accompanists changing with vocal music theme and emotional development. Accompaniment sense seems to be very significant. Performers grasp it sensibly. Naturally, this intuition is certainly based on daily rational hard training.

\section{Specific application strategy of balance theory in vocal music piano accompaniment}

\section{Appropriately dispose musical structure}

In the face of intricate melody lines, it is required to more clearly know what material is most important for piano and which part is most interesting for piano. Thus, piano accompanists should guide. For the decorations which are not so important, it is required to keep them in the background. Such problem that high pitch part at the tight hand of piano accompanists is matched with singers' melody often occurs. Generally, a large number of piano accompanists will blend in it involuntarily so as to create gorgeous melody line together with the singer. Of course, a taboo should be noticed: very rich harmony texture must be fused in instrumental ensemble, and repeated intensification of high pitch melody will certainly result in the conflict between piano accompanists and singers, and the trend of monotony and unbalance. Meanwhile, audiences will feel ear-piercing acoustically due to singleness. Therefore, piano accompaniment part should depart from singers' melody to the largest extent. It is required to make efforts to fill in and enrich harmony texture so as to give singers powerful support. Besides, a factor is often neglected by modern people, i.e. low pitch melody line. In fact, if a singer is performing graceful and touching melody, piano accompaniment will often generate and keep symmetrical low pitch melody. A part may be the same with high pitch melody. Vocal music piano accompaniment melody is exactly low pitch melody line that people may easily neglect. This melody line makes low pitch line without molding force as per harmonic movement and owns internationally advanced symmetrical balance with singers' high pitch melody line. Thus, if the range of the partner is higher, low pitch part should be further highlighted.

\section{Prudently apply the pedal}

An important function of the pedal is to mix color and render atmosphere. If damper pedal and soft pedal can be used reliably, this will provide timely help for handling vocal music works. However, once the pedal is misused or used improperly, the original purpose of the composer may be changed and an unsuccessful music works may be caused. Naturally, this is also a common problem affecting balance factor. Pedal application methods are not diversified, and full of resilience. When the pedal is applied, differentiation depth and different expressive force should be tried and experienced. Once large quantities of chords are stacked repeatedly, the performer must adopt thrilling pedal form to control the density of chords. This method can clean feculent strings. Of course, various harmonies may meet sometimes. At this moment, the performer should quickly change the pedal to ensure 
smooth transition with chords. At the special expressive force, the performer may use left pedal. At this moment, it is necessary to flexibly control the sound of pedal trampling and loosening without any seam. Once the left pedal is excessively used, extreme vague sound will be caused. This also violates the principle of pursuing clearness in balance. Pedal trace cannot be heard in high-level performance. The performer needs to show the consistency with fingers, rather than using the pedal. The performer may try to use the lines in the music score and connect the third joint of five fingers to achieve connection and coherent transmission.

\section{Adjust piano intensity as per cooperation object and works}

For piano accompanists, they need to cooperate with all kinds of instruments or singers. Different singers have huge differences. Each individual is different. The same mark must adopt different standards to adapt diverse music types. For the same strong mark, it should be stronger during Debussy's piano music works than that during performing Tchaikovsky's music works. The range has great differences for each instrument or voice. For every singer, a range is weaker than other ranges in low pitch zone, middle pitch zone or high pitch zone. For the same song, male singers and female singers have different strength in the same range. For example, with regard to piano accompaniment in the low pitch zone, originally normal and standard strong mark may be suitable for soprano, but not suitable for baritone. Meanwhile, in the same range, high pitch and low pitch of accompaniment are exactly opposite in the face of singers with different voice parts. So, to comply with detailed conditions of different types of singers, vocal music piano accompaniment personnel should adjust their volume as per actual conditions. Of course, the speed is also one of important factors influencing strength change. At the speed of adagio or andante, specific effects generated by the same mark is stronger than allegro or presto. In allegro, singers will reduce strength. In such case, if piano accompaniment continues to keep such standard strength, singers will certainly be submerged. Once the partner's speed become faster, piano accompanist needs to play more lightly.

\section{Notice differences between formal performance and rehearsal}

Once all factors influencing balance can achieve perfect clearness, the most critical factor is sound effect on the performance site. About this problem, two aspects need considering: whether the auditory sense is correct in the performance process. This is because it is very difficult to ensure clear auditory sense in the performance process. Once audiences more relax, the sound they hear further approach real effect. On the contrary, if audiences are nervous, the voice of the singer they hear will become low. The other problem is on-site effect. The partner will often walk on the stage to measure balance problem of sound effect. However, such measurement will make people generate imagination. Since the performance site is empty, once it is filled with audiences, the voice of the singer heard by the piano accompanist can amount to the voice heard by the audiences, because the singer standards back towards the accompanist and faces the vast audiences. In the balance theory of vocal music piano accompaniment, piano cover opening and closing most deserve noticing, but may be easily neglected. People worry the piano may surpass the singer in the volume or aura. So, generally, the piano cover should be opened a little or fully closed. After it is fully closed, it is like broadcaster's microphone covered by a cotton quilt so that the will cannot the clearly expressed. This violates balance principle as analyzed above. Once the piano cover is fully closed, only the volume is controlled. However, the information conveyed to the singer in piano accompaniment is also limited. Even the piano performer is also difficult to clearly distinguish the actual effect after the hammer knocks on the string and then reflects in the ear. At this time, piano accompaniment may easily deliberately amplify the volume in order to pinpoint the clearness. On the contrary, once the piano cover is opened fully, differential touch of piano player can be conveyed to the singer's ears through the piano cover so that string resonance and singer's resonance can be well fused to achieve perfect balance. Therefore, if the piano cover is closed due to volume problem, the effect will not be good. Instrumental ensemble balance needs to be achieved through multi-factor clearness rather than only the volume factor. 


\section{Conclusion}

In conclusion, although vocal music piano accompaniment is closely related to piano, vocal music and other main courses, it as a very important music art plays a very significant role in music teaching field. As socialist economy with Chinese characteristics has been improved and perfected in recent years, the demand for high-level piano accompaniment talents is also on the rise. Of course, under the general background of comprehensively deepening the reform, music teaching in most schools regard piano as an essential music instrument. More wide teaching activities in and outside schools propose new and higher requirements for piano accompaniment ability of music education workers. Vocal music piano accompaniment teaching should not rest on current accompaniment skills and superficial cognition of theoretical knowledge, but should be devoted to higher-level mastery of the essence of art. So, vocal music piano accompaniment needs to practically improve teaching quality in order to get a higher state.

\section{References}

[1] Zou yanzhuo, On contrast, balance and unification in vocal music piano accompaniment. The New Voice of Yue-fu (The Academic Periodical of Shenyang Conservatory of Music), 2009 (4).

[2] Zhang Xuliang, Strengthen service awareness for music education and enhance piano accompaniment ability training - study on piano accompaniment ability training mechanism for undergraduates majoring in musicology (education) in regular universities. People's Music, 2009 (5).

[3] Xu Yan, On contrast, balance and unification in vocal music piano accompaniment. Intelligence, 2014 (2).

[4] Huang Qibin, Discussion on contract in vocal music piano accompaniment - balance and unification. Music Space, 2014 (22). 